\title{
Superstructure of Uniaxially Oriented $\gamma$ Nylon 6 at Swelling Equilibrium in Solvent Mixtures
}

\author{
Zeyong ZHENG, Hideki KolZuMI, Shuichi NoJIMA,* \\ and Tamaichi ASHIDA \\ Department of Applied Chemistry, Faculty of Engineering, \\ Nagoya University, Nagoya 464-01, Japan
}

(Received July 11, 1989)

\begin{abstract}
The superstructure of uniaxially oriented $\gamma$ nylon 6 was investigated at swelling equilibrium in solvent-nonsolvent mixtures by means of small angle X-ray scattering. The parameters which characterize the superstructure, such as lamella thickness, long period, lateral size of the lamella, and spacing in the lateral direction, were evaluated based on a paracrystalline macrolattice model proposed by Wilke et al. These parameters were found to be independent of the swelling agent. The dependence of the parameters on the swelling degree was qualitatively similar to that previously observed on the swelling behavior with $\alpha$ nylon 6 , indicating that there is no significant effect of the crystal structure and superstructure on the swelling behavior of the nylon 6 .

KEY WORDS Swelling Equilibrium / $\gamma$ Nylon $6 /$ Small Angle X-Ray Scattering / Superstructure / Macrolattice Model/
\end{abstract}

Investigation on the swelling behavior of crystalline polymers is one of the powerful means to understand the superstructure of the polymers. The long period, alternate stacking of the lamella and the amorphous layer, of oriented crystalline polymers has been measured at swelling equilibrium by many authors using small angle X-ray scattering (SAXS) technique, ${ }^{1-5}$ and the change of the superstructure has been discussed as a function of swelling degree. In these studies, only part of the information about the superstructure included in the SAXS pattern has been used, because the model which described the details of superstructure in oriented crystalline polymers was not available. Fronk and Wilke et al. recently proposed ${ }^{6-8}$ a superstructure model for uniaxially oriented crystalline polymers based on a paracrystalline lattice model introduced by Hosemann et al. ${ }^{9}$ with which it is possible to evaluate the parameters in the model from a fit of the theoretical intensity to the experimental SAXS intensity.

We recently reported ${ }^{10}$ the swelling behavior of uniaxially oriented $\alpha$ nylon 6 studied with the SAXS technique, and evaluated the dependence of superstructure on the swelling degree $Q$. The long period (denoted by $a_{3}$ in our previous paper) and the spacing in the lateral direction $\left(a_{\mathrm{r}}\right)$ increased slightly with increasing $Q$, and the lateral size of lamella $\left(D_{1}\right)$ was invariable. The lamella thickness $(H)$ had a minimum at about $Q=12 \%$, which was attributable to the rearrangement of the amorphous polymer chain on the lamella surface with increasing $Q$.

In this paper, the swelling behavior of $\gamma$ nylon 6 is investigated. Combinations of the solvent and nonsolvent were the same as those used in the previous study of $\alpha$ nylon $6 .{ }^{10}$ The results are compared with each other, and the swelling behavior is discussed on the basis of

* To whom correspondence should be addressed. 
the crystal structure and superstructure.

\section{EXPERIMENTAL}

\section{Preparation of $\gamma$ Nylon 6}

The nylon 6 used in this study was kindly supplied by Toray Industry Inc. in the form of an unoriented sheet with a thickness of $0.5 \mathrm{~mm}$. Three strips, $3 \mathrm{~mm}$ wide and $30 \mathrm{~mm}$ long, were cut out from the original sheet and overlapped together with $85 \%$ aqueous formic acid. This sample was drawn up to 3 times in length at room temperature (cold drawing). The uniaxially oriented nylon 6 was annealed at $190^{\circ} \mathrm{C}$ under vacuum for $3 \mathrm{~h}$. The sample was further treated with iodine $\left(\mathrm{I}_{2}\right)$ potassium iodide (KI) aqueous solution for 10 days, followed by treating with sodium thiosulfate aqueous solution for 10 days to remove the $\mathrm{KI}$ and $\mathrm{I}_{2}$, and washed in fresh water for 7 days to remove the sodium thiosulfate. ${ }^{11}$ The sample was dried under vacuum at room temperature and stored in a dry place until use. Characteristic reflection with $\alpha$ nylon $6^{12,13}$ was not observed in a wide angle X-ray scattering (WAXS) pattern of this sample, indicating that uniaxially oriented nylon 6 with the crystallites of $100 \% \gamma$ form was produced in the sample.

\section{Systems and Equilibrium Swelling Degree}

Three solvent-nonsolvent mixtures were employed to produce swelling equilibrium between the solvent and the amorphous region of the $\gamma$ nylon 6, as shown in Table I. Benzyl alcohol can penetrate into the amorphous region of nylon 6 but never dissolves the

Table 1. Systems used in this study

\begin{tabular}{cccl}
\hline System & Polymer & Solvent & Non-solvent \\
\hline a & $\gamma$ Nylon 6 & Benzyl alcohol & Ethyl acetate \\
b & $\gamma$ Nylon 6 & Benzyl alcohol & Cyclohexanon \\
c & $\gamma$ Nylon 6 & Phenol $^{\text {a }}$ & $n$-Heptane
\end{tabular}

a Phenol is a relatively good solvent for nylon 6 , and thus low phenol concentrations up to $1 \%$ were used. crystalline region (lamella). Phenol is a rather good solvent and a swelling experiment was carried out at low concentrations up to $1 \%$ in the solvent-nonsolvent mixture. The nonsolvent (ethyl acetate or cyclohexanon) cannot penetrate into the amorphous region at all and regulates the swelling degree of the solvent through affinity between the solvent and nonsolvent. Equilibrium swelling degree $Q$ is defined as $Q=\left(W-W_{0}\right) / W_{0}$, where $W$ and $W_{0}$ represent the weights of swollen and dry $\gamma$ nylon 6 , respectively. It took 2 to 3 days for all samples investigated to reach the swelling equilibrium.

\section{Small Angle X-Ray Scattering (SAXS)}

\section{Measurement}

All SAXS patterns were obtained with a one-dimensional position sensitive proportional counter (PSPC) combined with a rotating anode X-ray generator (RIGAKU RU300 ) operated at $50 \mathrm{kV}$ and $100 \mathrm{~mA}$. This uniaxially oriented $\gamma$ nylon 6 produced the diffracted intensity maxima on the meridian (called two-point scattering), as schematically shown in Figure la. The intensity profiles along three sections $\mathrm{A}, \mathrm{B}$, and $\mathrm{C}$ were measured by rotating the sample in the plane perpendicular to the incident beam or by moving the PSPC along the meridian;

section $\mathrm{A}$, through the origin and along the meridian;

section B, pararell to the equator and through one of the intensity maxima on the meridian;

section $\mathrm{C}$, through the origin and forming an angle of $20^{\circ}$ with the meridian.

The typical intensity for each section is shown in Figure 1b. These intensity profiles are enough to characterize the two-point SAXS pattern observed. The intensity measured was corrected for the PSPC sensitivity and linearity, the absorption by the sample, and the background intensity.

The SAXS data were analysed based on a paracrystalline macrolattice model (see Figure 

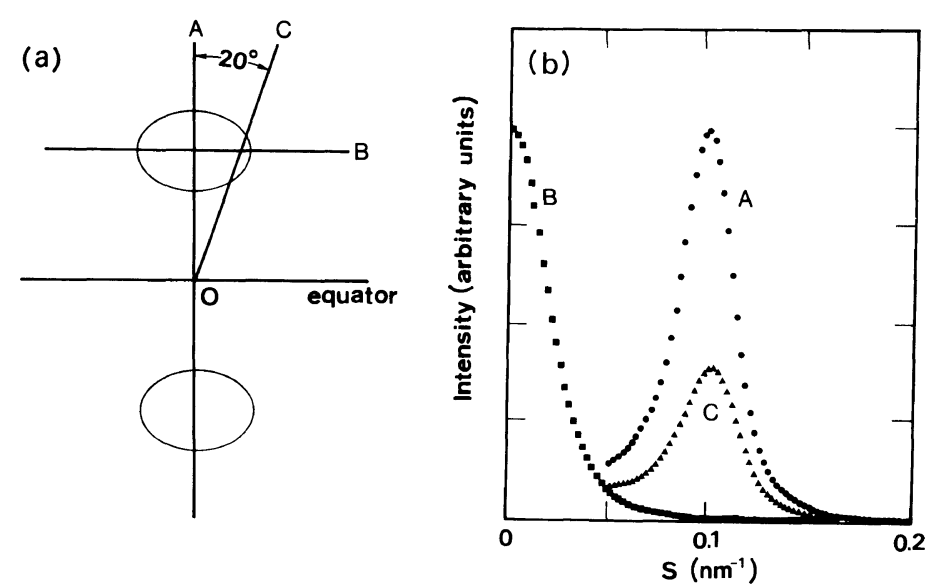

Figure 1. (a) Schematic illustration of the SAXS intensity from a uniaxially oriented $\gamma$ nylon 6 strip. The SAXS profile shows the intensity maxima on the meridian (two-point scattering). Only the intensity profiles along the sections $\mathrm{A}, \mathrm{B}$, and $\mathrm{C}$ are measured.

(b) Typical SAXS intensity profiles of sections A $(\boldsymbol{O}), \mathrm{B}(\boldsymbol{\square})$, and C ( $\mathbf{\Delta})$ for system b at $Q=26.8 \%$. The SAXS intensity at lower $\mathrm{s}$ for sections $\mathrm{A}$ and $\mathrm{C}$ is eliminated because of the beam stop.

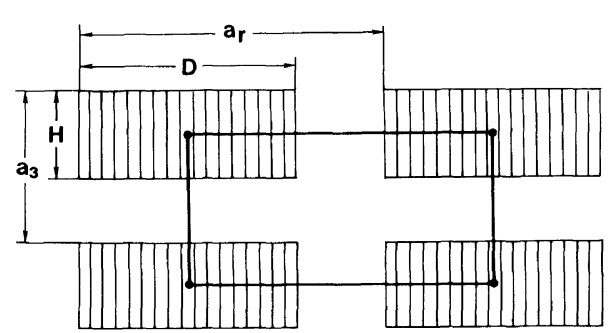

Figure 2. Schematic picture of the macrolattice model used in the calculation of the theoretical intensity. The square connecting the four lamellae represents a paracrystalline lattice introduced by Hosemann et al.

2) recently proposed by Fronk and Wilke. ${ }^{6-8}$ Parameters included in this model, lamella thickness (denoted by $H$ in Figure 2), long period $\left(a_{3}\right)$, lateral size of the lamella (denoted by $D$ instead of $D_{1}$ in our previous paper), and spacing in the lateral direction $\left(a_{\mathrm{r}}\right)$, were evaluated from the fit of the theoretical intensity to the experimental SAXS data as a function of the swelling degree. The details of the fit procedure were previously described. ${ }^{10}$

\section{RESULTS AND DISCUSSION}

\section{Swelling behavior}

Figure 3 shows the dependence of the swell-
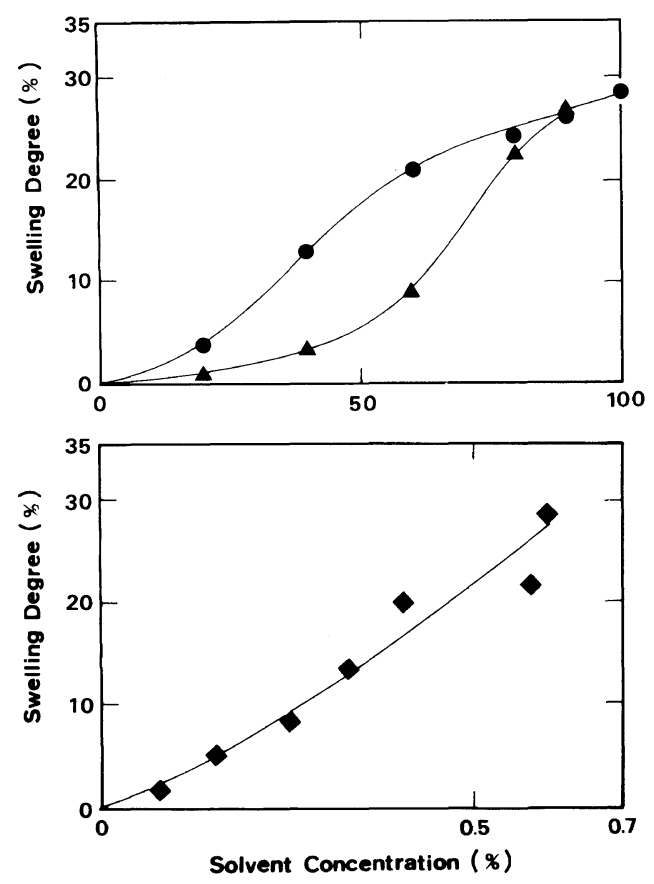

Figure 3. Swelling degree against the solvent concentration in the solvent-nonsolvent mixture. system a; $\boldsymbol{\Delta}$, system b; $\bullet$, system c.

ing degree $Q$ on the solvent concentration in the solvent-nonsolvent mixture for systems $a, b$, and c. In systems a and b, $Q$ shows a sigmoidal 
curve, though there is a significant difference in the solvent concentration at half the maximum swelling degree. In system c, $Q$ shows approximately a linear increase against the solvent concentration. The dependence of $Q$ on the solvent concentration observed for systems a, $\mathrm{b}$, and $\mathrm{c}$ is similar to that previously obtained for $\alpha$ nylon $6,{ }^{10}$ indicating that there is no effect of the crystal structure ( $\alpha$ form or $\gamma$ form) on the swelling behavior. The difference in the swelling behavior for systems a and $b$, where the interaction between the amorphous polymer and the solvent is comparable, can be interpreted in terms of the affinity difference between the solvent and the nonsolvent. ${ }^{10}$

It will be found later (Table II) that there is a large difference in the superstructure between the $\alpha$ nylon $6^{10}$ and the present $\gamma$ nylon 6 . This difference should accompany the change of chain conformation in the amorphous region, because the conformation is governed by the average chain length traversing through the amorphous region, and this chain length is strongly influenced by the size or thickness of the amorphous region. When Figure 3 is compared with Figure 3 of ref 10, it is found that the swelling behavior observed against solvent concentration is not influenced by the superstructure in the sample, though the maximum swelling degree $Q_{\max }$ differs significantly (i.e., $Q_{\max } \sim 20 \%$ for $\alpha$ nylon 6 and $Q_{\max } \sim 30 \%$ for $\gamma$ nylon 6). This indicates that the swelling degree strongly depends on the conformation change of polymer chain in the amorphous

Table II. Comparison of superstructural parameters between dry $\alpha$ nylon 6 annealed at $196^{\circ} \mathrm{C}$ and $\gamma$ nylon 6 annealed at $190^{\circ} \mathrm{C}$

\begin{tabular}{ccc}
\hline & $\alpha$ Nylon 6 & $\gamma$ Nylon 6 \\
\hline$a_{3}(\mathrm{~nm})$ & 10.12 & 8.31 \\
$a_{\mathrm{r}}(\mathrm{nm})$ & 18.14 & 13.72 \\
$H(\mathrm{~nm})$ & 5.64 & 4.53 \\
$D(\mathrm{~nm})$ & 15.63 & 13.69 \\
Crystallinity* & 0.414 & 0.543 \\
\hline
\end{tabular}

a Calculated from $a_{3}, a_{\mathrm{r}}, H$, and $D$. region, but the swelling behavior against the solvent concentration does not.

\section{Superstructure}

Figure 4 shows a typical example of the fit of the theoretical intensity to the experimental SAXS data for each section. A similar fit was achieved for other SAXS data measured. From this fit, lamella thickness (denoted by $H$ in Figure 2), long period $\left(a_{3}\right)$, lateral size of the lamella $(D)$, and spacing in the lateral direction $\left(a_{\mathrm{r}}\right)$ could be evaluated. Table II shows a comparison of the parameters which characterize the superstructure of dry $\alpha$ nylon 6 and $\gamma$ nylon 6 (before swelling). The components of the repeat unit, the lamella and the amorphous layer, of $\gamma$ nylon 6 are smaller in size, and the total.crystallinity is higher than that of $\alpha$ nylon 6.

Figure 5a and $\mathrm{b}$ show the $Q$ dependence of $H$ and $a_{3}$, where the open symbols refer to $\alpha$ nylon 6 and closed to $\gamma$ nylon 6 . In both systems, the $Q$ dependence of $H$ in $\gamma$ nylon 6 is similar to that in $\alpha$ nylon 6 ; first $H$ decreases slightly with $Q$ and begins to increase, resulting in a minimum at about $Q=12 \%$, and finally levels off at a constant value. Though the initial value of $H$ in $\gamma$ nylon 6 is quite

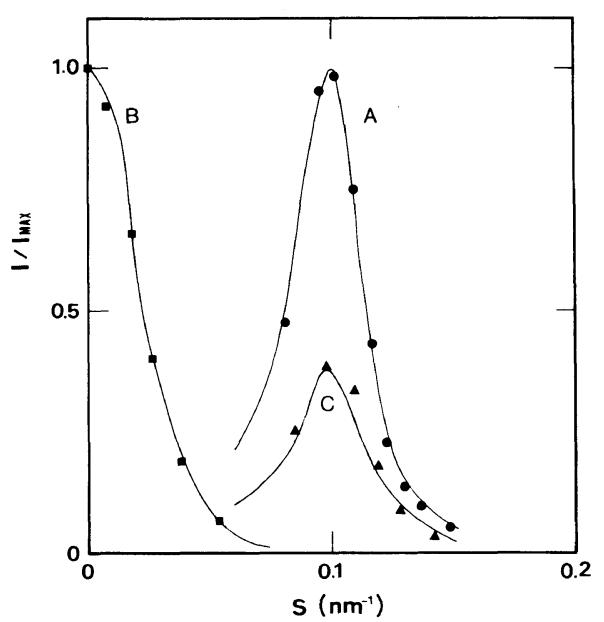

Figure 4. Typical example of the fit between theoretical intensity and experimental SAXS intensity for system b at $Q=26.8 \%$. 


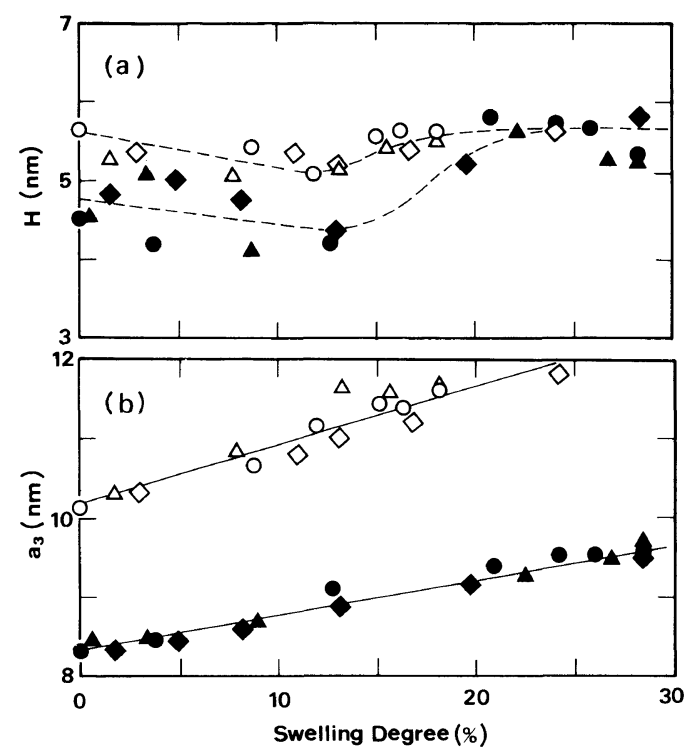

Figure 5. The dependence of $H$ (upper figure) and $a_{3}$ (lower figure) on the swelling degree. Open symbols are for $\alpha$ nylon 6 previously obtained, and closed symbols $\gamma$ nylon 6 . $\bigcirc, \diamond$, system a; $\triangle, \mathbf{\Delta}$, system b; $\diamond, \diamond$, system c.

smaller than that in $\alpha$ nylon $6, H$ at large $Q$ is almost the same, leading to variation in $H$ of the samples with $\gamma$ nylon 6 more enhanced. The $Q$ dependence of $H$ was previously explained by rearrangement of the amorphous chains just coming out from the lamella. The increase in $H$ at large $Q$ was also explained successfully assuming the stretching of tie molecules in the amorphous layer between lamellae to form a pseudocrystalline region on the lamella surface. The crystallinity of the present $\gamma$ nylon 6 is higher than that of the $\alpha$ nylon 6 (Table II), so that the amorphous region per unit volume of the $\gamma$ nylon 6 accommodates more solvent than that of the $\alpha$ nylon 6 at the same $Q$. This indicates that the amorphous chain of the $\gamma$ nylon 6 incurs stronger extension, resulting in the pseudocrystalline layer thicker.

The $Q$ dependence of $a_{3}$ is shown in Figure $5 b$, where all data points for systems with $\alpha$ nylon 6 arrange roughly on one line and those for $\gamma$ nylon on another line. The difference in

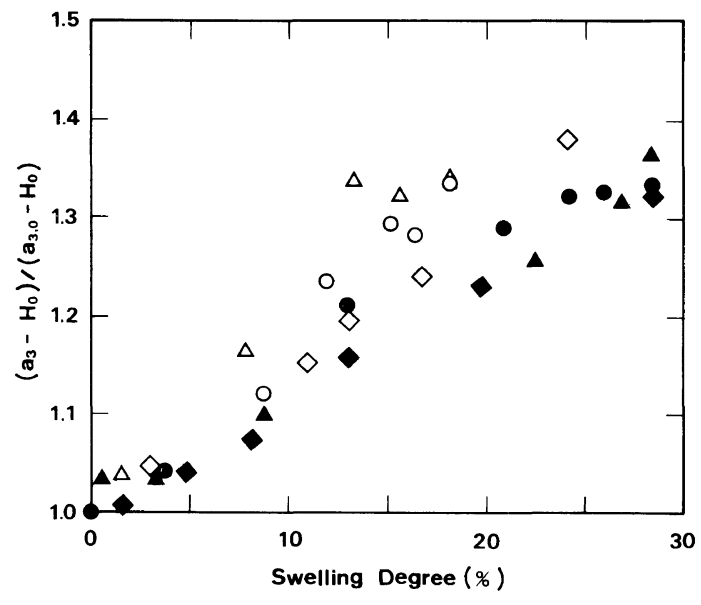

Figure 6. Reduced thickness of amorphous region between lamellae, $\left(a_{3}-\mathrm{H}_{0}\right) /\left(a_{3.0}-\mathrm{H}_{0}\right)$ against swelling degree. For the symbols, see Figure 5.

the absolute value between two lines arises from the superstructure in the samples before swelling. The slope of the line for $\gamma$ nylon 6 is 0.047 ( $\mathrm{nm} / \mathrm{swelling}$ degree $)$, which is smaller than that for $\alpha$ nylon $6,0.077(\mathrm{~nm} /$ swelling degree). To understand the difference in the slope, it is necessary to take into account the thickness of the amorphous layer before swelling. If the swelling agent (solvent) is assumed to be completely accommodated in the amorphous region without being absorbed by the lamella, which is one of the conclusions of our previous paper, the slope should depend on the initial thickness of the amorphous layer. Therefore, it is necessary to compare both results in terms of the swelling rate, the $Q$ dependence of the amorphous layer thickness per original thickness. The net thickness of the amorphous layer at swelling equilibrium can be estimated by subtracting $H_{0}$ from $a_{3}$ to exclude the effect of chain rearrangement on the lamella. Figure 6 shows the $Q$ dependence of the reduced amorphous layer $\left(a_{3}-\mathrm{H}_{0}\right)$ $\left(a_{3,0}-\mathrm{H}_{0}\right)$, where the subscript 0 stands for the initial value. The two sets of data points shown in Figure $5 \mathrm{~b}$ become closer to each other in Figure 6 and seem to make one line with a common slope, though there is small incon- 
sistency between the two sets of data points. This systematic difference is probably due to the approximation in calculating the reduced amorphous layer, in addition to the estimation error of the parameters in the fit process. However, if we neglect this small difference, it is likely that the swelling behavior per unit length of the amorphous layer is independent of the crystal structure and the superstructure. It will be found later that the amorphous region in the lateral direction also accommodates the swelling agent, and this amount per unit length at same $Q$ is almost the same as the amount accommodated by the amorphous layer between lamellae.

Figure 7 shows the $Q$ dependence of the lateral size of the lamella $D$ and the lateral distance $a_{\mathrm{r}}$, where $D$ is independent of the swelling degree and maintains it's initial value over a wide range of $Q$. This means that the lamella is not attacked by the swelling agent in the lateral direction. The $a_{\mathrm{r}}$ of $\alpha$ nylon 6 increases slightly with $Q$, but $a_{\mathrm{r}}$ of $\gamma$ nylon 6 seems to increase only slightly. The fit procedure is presumably responsible for the scat-

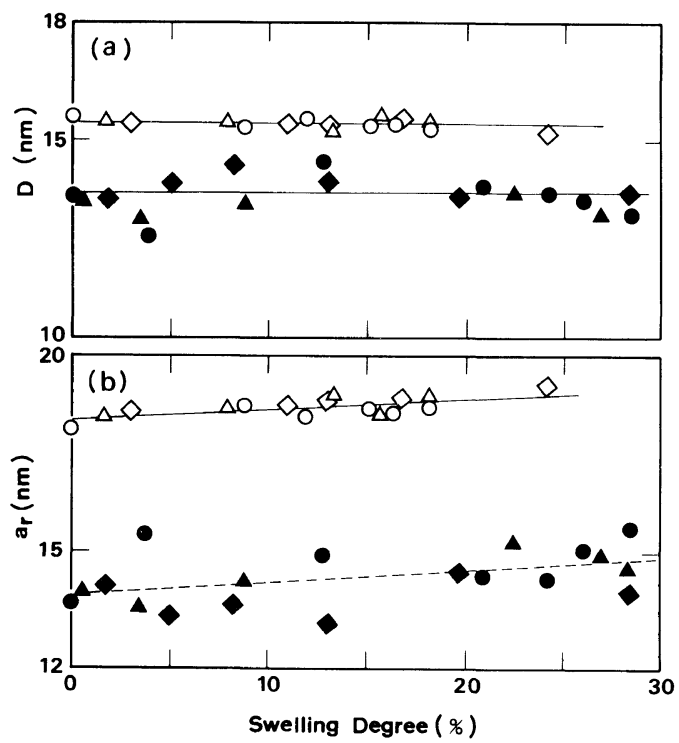

Figure 7. The dependence of $D$ (upper figure) and $a_{\mathrm{r}}$ (lower figure) on the swelling degree. For the symbols, see Figure 5. ter of the data points in Figure 7. The increase of $a_{\mathrm{r}}$ indicates the swelling of the amorphous region by the solvent in the lateral direction. In $\alpha$ nylon 6 , the swelling rate, the $Q$ dependence of the thickness of the amorphous layer per unit length, is in accordance with that obtained for $a_{3}$ in Figure 6. The difference between $a_{\mathrm{r}}$ and $D$ in $\gamma$ nylon 6 , however, is quite small as shown in Table II, and even if the swelling takes place in this amorphous region, it is hard to detect appreciable increase in $a_{\mathrm{r}}$.

\section{Comparison between Microscopic and Macroscopic Swelling Degrees}

The macroscopic swelling degree $Q$, estimated from the weights of the sample before and after swelling, has been used in the discussion. A comparison of the $Q$ with the microscopic swelling degree $Q^{\prime}$, which can be calculated from the parameters evaluated through the fit procedure, usually gives information about some other superstructure in the sample. Dosiere et al., for example, observed ${ }^{3}$ a significant difference between $Q$ and $Q^{\prime}$ by a factor of 2 in a doubly oriented nylon 11 , and found the existence of amorphous domains possessing no crystallite at all.

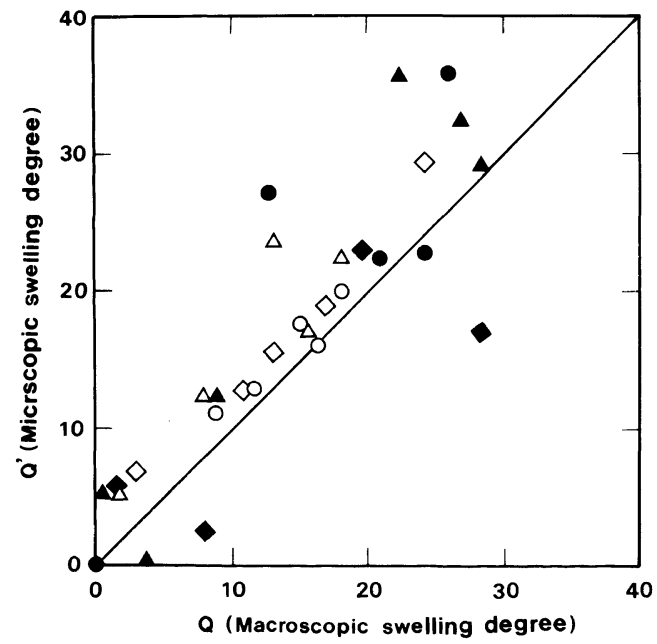

Figure 8. Comparison between the macroscopic swelling degree $Q$ and the microscopic swelling degree $Q^{\prime}$. For the symbols see Figure 5. 
The microscopic swelling degree $Q^{\prime}$ is defined assuming the additivity of the volumes of the solvent and the amorphous region,

$$
Q^{\prime}=\left(V-V_{\mathrm{c}}-V_{\mathrm{a}}\right) \rho_{\mathrm{s}} /\left(\rho_{\mathrm{c}} V_{\mathrm{c}}+\rho_{\mathrm{a}} V_{\mathrm{a}}\right)
$$

where $\rho_{\mathrm{c}}, \rho_{\mathrm{a}}$, and $\rho_{\mathrm{s}}$ stand for the densities of the crystal, the amorphous region of nylon 6 , and the solvent, respectively, and $V_{\mathrm{c}}$ and $V_{\mathrm{a}}$ are the volumes of the crystal and the amorphous region in a macrolattice cell of the nylon 6 before swelling (see Figure 2). $V$ is the cell volume at the swelling equilibrium. The following densities were used in the calculation; $\rho_{\mathrm{s}}=1.049\left(\mathrm{~g} \mathrm{~cm}^{-3}\right)$ for benzyl alcohol, $\rho_{\mathrm{s}}=$ $1.071\left(\mathrm{~g} \mathrm{~cm}^{-3}\right)$ for phenol, $\rho_{\mathrm{c}}=1.235\left(\mathrm{~g} \mathrm{~cm}^{-3}\right)$ for the crystal of the $\alpha$ nylon $6,{ }^{12} \rho_{\mathrm{c}}=1.190$ $\left(\mathrm{g} \mathrm{cm}^{-3}\right)$ for the crystal of the $\gamma$ nylon $6,{ }^{14}$ and $\rho_{\mathrm{a}}=1.091\left(\mathrm{~g} \mathrm{~cm}^{-3}\right)$ for the amorphous region of the nylon. ${ }^{15}$

Figure 8 shows a comparison of $Q$ and $Q^{\prime}$, where the solid line stands for $Q=Q^{\prime}$. If there are large domains in the sample where no crystallite is observed, the data points should be located far below the solid line, as is the case with nylon 11 observed by Dosiere. ${ }^{3}$ Though the data points in Figure 8 are somewhat scattered at large swelling degree, the microscopic swelling degree $Q^{\prime}$ is comparable to the macroscopic swelling degree $Q$. This indicates that there is no large amorphous domain and the samples used in the present study ( $\alpha$ nylon 6 and $\gamma$ nylon 6) are filled with crystalline regions consisting of alternate stackings of lamella and amorphous layer.

\section{CONCLUSIONS}

The swelling behavior of uniaxially oriented $\gamma$ nylon 6 was investigated for three combinations of solvent-nonsolvent mixtures by means of small angle X-ray scattering (SAXS). The SAXS intensity was analysed with a paracrystalline macrolattice model recently proposed by Wilke et al. Parameters which characterize the superstructure were evaluated, and compared with those previously evaluated in the swelling study with $\alpha$ nylon 6 .

1. The superstructure was influenced by the equilibrium swelling degree but independent of the swelling agent used.

2. Each parameter was significantly dependent on the superstructure before swelling. The swelling behavior of the parameters was similar between $\alpha$ nylon 6 and $\gamma$ nylon 6 .

3. The lamella thickness had a minimum with changing the swelling degree, which is more enhanced in $\gamma$ nylon 6 than in $\alpha$ nylon 6 .

4. The macroscopic swelling degree $Q$, obtained from the weights of the sample before and after swelling, is comparable with the microscopic swelling degree $Q^{\prime}$, evaluated from the superstructure parameters. This indicates that there is no large amorphous domain in the samples investigated.

Acknowledgement. We should like to thank the staff of the Workshop for Experimentation and Practice, Faculty of Engineering, Nagoya University, for making a vacuum line to treat the samples at high temperature.

\section{REFERENCES}

1. Y. Udagawa and A. Keller, J. Polym. Sci. A-2, 9, 437 (1971).

2. G. V. Fraser, A. Keller, E. J. George, and D. Dreyfuss, J. Macromol. Sci.-Phys., B16, 295 (1979).

3. M. Dosiere, J. Mater. Sci., 18, 1941 (1983).

4. M. Dosiere and J. J. Point, J. Polym. Sci., Polym. Phys. Ed., 22, 749 (1984).

5. I. R. Harrison, T. J. Weaver, and J. Runt, Polym. Commun., 26, 244 (1985).

6. K. Gottlicher, W. Fronk, and W. Wilke, Coll. Polym. Sci., 261, 126 (1985).

7. W. Fronk and W. Wilke, Coll. Polym. Sci., 263, 97 (1985).

8. W. Fronk and W. Wilke, J. Polym. Sci., Polym. Phys. Ed., 24, 839 (1986).

9. R. Hosemann and S. Bagchi, "Direct Analysis" of Diffraction by Matter," North-Holland, Amsterdam, 1962.

10. Z. Zheng, S. Nojima, T. Yamane, and T. Ashida, Polym. J., 21, 65 (1989).

11. K. Hoashi and R. D. Andrews, J. Polym. Sci., C38. 387 (1972). 


\section{Z. ZHENG et al.}

12. D. R. Holmer, C. W. Bunn, and D. J. Smith, J. Polym. Sci., 17, 159 (1955).

13. H. Arimoto, J. Polym. Sci., A2, 2283 (1964).
14. K. H. Illers, H. Harberkorn, and P. Simak, Makromol Chem., 158, 285 (1972).

15. K. H. Illers, Makromol Chem., 179, 497 (1978). 